\title{
Factors Related to Insomnia in Adult Patients with Tinnitus and/or Hyperacusis: An Exploratory Analysis
}

DOI: $10.3766 /$ jaaa. 18020

\author{
Hashir Aazh* \\ David M. Baguley†‡ \\ Brian C. J. Moore§
}

\begin{abstract}
Background: People with tinnitus and/or hyperacusis often experience insomnia. However, it is unclear what factors are most strongly associated with insomnia.
\end{abstract}

Purpose: To explore factors related to insomnia in patients with tinnitus and/or hyperacusis.

Research Design: This was a retrospective study using multiple-regression analysis.

Study Sample: Data were assessed for 444 consecutive patients who sought help concerning their tinnitus and/or hyperacusis from a specialist audiology center in the UK National Health Service. The average age of the patients was 54 years (standard deviation $=15$ years).

Data Collection and Analysis: The results of audiological tests and self-report questionnaires were gathered retrospectively from the records of the patients. Multiple-regression analysis was used to assess the relationship between insomnia and other variables.

Results: Sixty-nine percent of patients with tinnitus experienced some form of insomnia as measured via the Insomnia Severity Index (ISI). A multiple-regression model showed that ISI scores were significantly associated with depression scores measured via the depression subscale of the Hospital Anxiety and Depression Scale (HADS) (regression coefficient $[b]=0.44, p<0.001$ ), tinnitus annoyance measured via the visual analog scale (VAS) $(b=0.49, p=0.001)$, anxiety measured via the anxiety subscale of the HADS $(b=0.21, p=0.012)$, and tinnitus handicap measured via the Tinnitus Handicap Inventory $(b=$ $0.07, p<0.001)$. The model explained $45 \%$ of the variance in ISI scores. VAS scores for tinnitus loudness, hyperacusis-related measures, hearing thresholds, age, and gender were not significantly related to insomnia.

Conclusions: The prevalence of insomnia in patients with tinnitus is high. Both tinnitus-related factors and psychological factors are related to the severity of insomnia. Hyperacusis is not significantly associated with insomnia.

Key Words: anxiety, depression, hyperacusis, insomnia, tinnitus

Abbreviations: BDI-II = Beck Depression Inventory II; HADS = Hospital Anxiety and Depression Scale; $\mathrm{HQ}=$ Hyperacusis Questionnaire; HTs = hearing thresholds; ISI = Insomnia Severity Index; OR = odds ratio; PSQI = Pittsburgh Sleep Quality Index; RIS = Regensburg Insomnia Scale; SAS = Self-Rating Anxiety Scale; SD = standard deviation; SDS = Self-Rating Depression Scale; $\mathrm{THI}=$ Tinnitus Handicap Inventory; TQ = Tinnitus Questionnaire; TRQ = Tinnitus Reaction Questionnaire; ULL = uncomfortable loudness level; ULLmin = average ULL at $0.25,0.5,1,2,4$, and $8 \mathrm{kHz}$ for the ear with the lower average ULL; VAS = visual analog scale

*Audiology Department, Royal Surrey County Hospital NHS Foundation Trust, Guildford, UK; †NIHR Nottingham Biomedical Research Centre, Nottingham, UK; $\$$ Otology and Hearing, Division of Clinical Neuroscience, School of Medicine, University of Nottingham, Nottingham, UK; §Department of Experimental Psychology, University of Cambridge, Cambridge, UK

Corresponding author: Hashir Aazh, Tinnitus and Hyperacusis Therapy Specialist Clinic, Audiology Department, Royal Surrey County Hospital, Guildford GU2 7XX, UK; Email: hashir.aazh@nhs.net 


\section{INTRODUCTION}

$\mathrm{T}$ innitus is the sensation of sound without any external acoustic sound source. Hyperacusis is a term used to describe intolerance to everyday sounds that causes significant distress and impairment in social, occupational, recreational, and other day-today activities (Aazh et al, 2016). Tinnitus and hyperacusis commonly occur together: approximately $90 \%$ of patients with a primary complaint of hyperacusis experience tinnitus, and $40 \%$ of those with a primary complaint of tinnitus experience hyperacusis (Baguley, 2018).

Tinnitus is also commonly associated with insomnia. Tyler and Baker (1983) reported that $57 \%$ of patients who were members of a tinnitus support group ( $\mathrm{n}=$ 97) reported having problems getting to sleep because of their tinnitus. Meikle et al (1984) reported that for patients experiencing tinnitus $(n=1,219)$, the incidence of sleep disturbances increased as the self-reported tinnitus severity increased. Folmer and Griest (2000) reported that the severity of tinnitus as measured via the Tinnitus Severity Index (Meikle et al, 1984) and via self-reported tinnitus loudness on a 1-10 scale was associated with the severity of sleep disturbances as measured via a single question asking whether tinnitus interfered with sleep. Miguel et al (2014) reported that scores on the Tinnitus Reaction Questionnaire (TRQ) (Wilson et al, 1991) were correlated $(r=0.62)$ with scores on the Insomnia Severity Index (ISI; Bastien et al, 2001) ( $\mathrm{n}=117)$. Schecklmann et al (2015) analyzed data for 182 patients with chronic tinnitus and found that scores on the Tinnitus Questionnaire (TQ) (Hallam et al, 1988) were correlated ( $r=$ 0.558 ) with scores on the Regensburg Insomnia Scale (RIS) (Cronlein et al, 2013). Cronlein et al (2016) used a regression analysis and reported that the somatic complaints subscale of the TQ (the subscale concerned with the presence of ear pain and muscle tension) and the emotional distress subscale of the TQ significantly predicted the severity of insomnia measured via the RIS $(n=173)$. However, their model predicted only $30 \%$ of the variance in RIS scores. Recent studies suggest that more than $70 \%$ of patients seeking help for tinnitus and/ or hyperacusis present with symptoms of insomnia (Schecklmann et al, 2015; Aazh et al, 2016).

The factors leading to insomnia for people with tinnitus remain somewhat unclear. From the clinical experience of the first author, a common concern expressed by patients is that although they can cope with the current loudness of their tinnitus, one of their fears is that if their tinnitus gets louder, it may stop them sleeping at night, leading to a constant state of fatigue that they would not be able to cope with. Andersson and Edvinsson (2008) pointed out that fear of the consequences of tinnitus is one of the main features of tinnitus sufferers. In the studies described previously, the possible role of anxiety and depression was not taken into account.

$\mathrm{Xu}$ et al (2016) conducted regression analyses to assess whether, for patients with tinnitus $(\mathrm{n}=543)$, sleep disturbances measured via the Pittsburgh Sleep Quality Index (PSQI) (Buysse et al, 1989) were related to anxiety and depression measured via the Self-Rating Anxiety Scale (SAS) (Zung, 1971) and the Self-Rating Depression Scale (SDS) (Zung, 1974). They reported significant associations between two measures of sleep problems, the time needed to get to sleep and day time dysfunction, and SAS and SDS scores. However, other aspects of sleep measured via the PSQI, such as habitual sleep efficiency, sleep disturbances, subjective sleep quality, sleep duration, and use of sleep medication, were not significantly associated with SAS and SDS scores. Xu et al (2016) did not include tinnitus-related measures in their model.

The models created by Cronlein et al (2016) and Xu et al (2016) did not take into account possible intervening factors. Doing so can markedly change regression coefficients (Kutner et al, 2004). Therefore, it is important to adjust any regression model to take into account the possible influence of other variables. Aazh et al (2017) assessed factors related to insomnia measured via the ISI (Bastien et al, 2001) among older patients with tinnitus and/or hyperacusis $(\mathrm{n}=151)$. They included scores for the Tinnitus Handicap Inventory (THI) (Newman et al, 1996) and the Hospital Anxiety and Depression Scale (HADS; Zigmond and Snaith, 1983) in their regression model. They found that higher scores on the ISI were associated with higher (worse) scores on the depression subscale of the HADS ( $p=$ 0.007 ). ISI scores were not significantly predicted by scores for the anxiety subscale of the HADS or by THI scores. However, their model explained only $32 \%$ of the variance in the ISI scores. Also, as insomnia is prevalent among older people whether or not they have tinnitus (Foley et al, 1995), it is not clear whether the model would be applicable to younger people.

The relationship between insomnia and hyperacusis is unclear. Hebert and Carrier (2007) assessed whether PSQI scores were related to tinnitus distress measured via the TRQ, hyperacusis handicap measured via the hyperacusis questionnaire (HQ) (Khalfa et al, 2002), and depression measured via the Beck Depression Inventory II (BDI-II) (Steer et al, 1997). They included 51 patients with tinnitus and 51 patients with no tinnitus. PSQI scores were associated with HQ and BDI-II scores, but not with average hearing thresholds (HTs) or TRQ scores for the group of patients with tinnitus. Their regression model explained $43 \%$ of the variance in PSQI scores. However, their sample had a mean age of 68 years. As mentioned previously, insomnia is prevalent among older people, so it is not clear whether the relationship found between insomnia and hyperacusis would occur for younger people. 
Fioretti et al (2013) reported that among 37 consecutive patients with tinnitus, $30 \%$ reported having both hyperacusis and sleep disorders. The presence of hyperacusis and sleep disorders was diagnosed based on patients' answers to the following questions: "Do you have a problem tolerating sounds because they often seem much too loud?" "Do you have difficulty falling asleep, waking in the night or too early in the morning?" They did not assess whether sleep disorders were more prevalent among patients with hyperacusis than among those with tinnitus only. Nordin and Nordin (2016) reported that $313 / 8,520$ people sampled from general population answered yes to the question "Do you have a hard time tolerating everyday sounds that you believe most other people can tolerate?" The incidence of sleep disturbances measured using the DSM-IV diagnostic criteria (American Psychiatric Association, 2000) for insomnia was significantly higher for this group than for those who answered no to the question. Baliatsas et al (2016) reported that for people from the general population who selected "strongly agree" on a 5-point scale from "strongly agree" to "strongly disagree" for the statement "I am sensitive to noise" ( $\mathrm{n}=722 / 5,806)$, the odds ratio (OR) of sleep problems as measured via the Sleep Quality Scale (Visser et al, 1978) was 1.94 (95\% confidence interval: 1.63-2.32) relative to those who chose options other than "strongly agree." Park et al (2017) assessed noise sensitivity for a sample of the general population ( $\mathrm{n}=1,836)$ via a single question with responses on an 11-point Likert scale: 0 indicated the lowest sensitivity and 10 indicated the highest sensitivity. They reported that the OR of insomnia, assessed using the ISI, was 2.05 for individuals with high noise sensitivity (scores 6-10) relative to those with low noise sensitivity (scores 0-5). They also reported that the OR of an abnormal score on the ISI was 2.08 after taking into account the effects of age, gender, income, and general health. However, they did not include measures of anxiety, depression, or tinnitus in their model. Thus, the influence of hyperacusis per se on insomnia remains unclear.

Given the high prevalence of insomnia among patients with tinnitus and/or hyperacusis, and the impact of insomnia on the general quality of life (Lasisi and Gureje, 2011), it is important to explore the factors that contribute to insomnia among patients with tinnitus and/or hyperacusis. The aim of this study was to explore factors associated with insomnia for patients who sought help with regard to their tinnitus and/or hyperacusis from an audiology clinic in the UK National Health Service.

\section{METHOD}

\section{Study Design and Patients}

This was a retrospective study conducted at the Tinnitus and Hyperacusis Therapy Specialist Clinic, Royal
Surrey County Hospital, Guildford, United Kingdom. Data were included for 444 consecutive patients who attended the clinic from January 2013 to January 2016 and who completed self-report questionnaires and audiological examinations. The average age of the patients was 54 years (standard deviation [SD] = 15 years). Forty-nine percent were male.

\section{Audiological Investigations}

Pure-tone audiograms were measured using the procedure recommended by the BSA (2011a). The severity of hearing loss was categorized based on the values of the pure-tone average over the frequencies $0.25,0.5$, 1,2 , and $4 \mathrm{kHz}$, as recommended by the BSA (2011a): mild (20-40 dB HL), moderate (41-70 dB $\mathrm{HL})$, severe (71-95 dB HL), and profound (>95 dB HL). Uncomfortable loudness levels (ULLs) were measured following the BSA recommended procedure (BSA, 2011b). When the average ULL at $0.25,0.5,1,2,4$, and 8 $\mathrm{kHz}$ for the ear with the lower average ULL, which is called ULLmin, was $\leq 77 \mathrm{~dB}$ HL, hyperacusis was deemed to be present (Aazh and Moore, 2017). The criterion for diagnosing severe hyperacusis was taken as a ULL of $30 \mathrm{~dB}$ HL or less for at least one of the measured frequencies, $0.25,0.5,1,2,3,4,6$, and $8 \mathrm{kHz}$, for at least one ear (Aazh and Moore, 2018).

\section{Assessment of Insomnia}

Insomnia was assessed using the ISI, which comprises seven items that assess the severity of sleep difficulties and their effect on the patient's life. Each item is rated on a scale from 0 to 4 and the total score ranges from 0 to 28 . Scores from 0 to 7 indicate no clinically significant insomnia, scores from 8 to 14 indicate slight insomnia, scores from 15 to 21 indicate moderate insomnia, and scores from 22 to 28 indicate severe insomnia (Bastien et al, 2001).

\section{Assessment of Tinnitus and Hyperacusis Handicap}

Tinnitus handicap was assessed using the THI, which has 25 items. The response choices are "no" $(0$ points), "sometimes" ( 2 points), and "yes" (4 points). The overall score ranges from 0 to 100 . Scores from 0 to 16 indicate no handicap, scores from 18 to 36 indicate mild handicap, scores from 38 to 56 indicate moderate handicap, and scores from 58 to 100 indicate severe handicap (Newman et al, 1996).

Hyperacusis handicap was assessed using the $\mathrm{HQ}$, which comprises 14 items. The response choices are "no" ( 0 points), "yes, a little" ( 1 point), "yes, quite a lot" ( 2 points), and "yes, a lot" (3 points). The overall score ranges from 0 to 
42. Scores above 22 were taken as indicating hyperacusis handicap (Aazh and Moore, 2017).

\section{Self-Reported Tinnitus Loudness, Annoyance, and Effect on Life}

The visual analog scale (VAS) was used to assess the self-reported loudness and annoyance of tinnitus and the effect of tinnitus on the patient's life. VAS scores are ratings on a scale from 0 to 10 . The VAS score for the loudness of tinnitus was assessed by asking the patient to rate the loudness of tinnitus during their waking hours over the last month (It was explained that 0 corresponds to no tinnitus being heard and 10 is the loudest that they can imagine). The VAS score for annoyance induced by the tinnitus was assessed by asking the patient to rate their subjective perception of annoyance on average during the last month (it was explained that 0 corresponds to no annoyance and 10 is the most annoying thing that can possibly happen). The VAS score for the impact of tinnitus on their life was assessed by asking the patient to rate the effect of tinnitus on their life during the last month (it was explained that 0 corresponds to no effect and 10 is an extreme effect).

\section{Assessment of Anxiety and Depression}

Anxiety and depression were evaluated using the HADS, which consists of 14 items, each rated from 0 to 3 according to the severity experienced. Eight items require reversed scoring, after which anxiety and depression subscale totals can be obtained. The total score for each subscale ranges from 0 to 21 . Scores from 0 to 7 are classified as normal, scores from 8 to 10 are classified as borderline abnormal, and scores from 11 to 21 are classified as abnormal (Zigmond and Snaith, 1983).

\section{Ethical Approval}

This study was approved by the South West Cornwall and Plymouth Research Ethics Committee.

\section{Data Analysis}

The data were anonymized before statistical analysis. Descriptive statistics (means and SDs) for the characteristics of the patients, audiological assessments, and scores for the self-report questionnaires were calculated. The scores were all approximately normally distributed. Pearson correlation tests and $\chi^{2}$ tests were used to assess the relationships between different measures and $t$-tests were used to assess mean differences. The $p$-value required for statistical significance was set at $p<0.05$. The variables that had significant correlations with ISI scores were included in a regression model to examine whether the variables significantly influenced the ISI score. A stepwise linear multiple regression model was created to predict the ISI score, beginning with a full model that included all variables. Then, variables were removed to assess whether their inclusion significantly affected the goodness of fit. The STATA program (version 13) was used for statistical analyses.

\section{RESULTS}

$\Upsilon$ he mean scores and SDs for the ISI, HADS, THI, 1 VAS, and HQ are shown in Table 1. Based on the scores for the ISI, 31\% (137) of patients did not have insomnia, 30\% (131) had mild insomnia, 28\% (122) had moderate insomnia, and 12\% (54/444) had severe insomnia. Based on scores for the THI, $12 \%$ of patients (52) had no tinnitus handicap, 32\% (141) had a mild tinnitus handicap, 24\% (105) had a moderate tinnitus handicap, and $33 \%$ (146) had a severe tinnitus handicap. For the anxiety subscale of the HADS, $41 \%$ (181) of patients had normal scores, 23\% (102) had borderline abnormal scores, and 36\% (161) had abnormal scores. For the depression subscale of the HADS, 68\% (301) of patients had normal scores, $14 \%$ (63) had borderline abnormal scores, and 18\% (80) had abnormal scores. Based on scores for the $\mathrm{HQ}, 32 \%$ (141) of patients had hyperacusis.

The means and SDs of the HTs and ULLs for each ear and each frequency are shown in Table 2. The mean pure-tone average across the frequencies $0.25,0.5,1$, 2 , and $4 \mathrm{kHz}$ and across ears was $20 \mathrm{~dB} \mathrm{HL}$ (SD = $13 \mathrm{~dB}$ ). The mean of the average ULL across 0.25 , $0.5,1,2,4$, and $8 \mathrm{kHz}$ and across ears was $83 \mathrm{~dB} \mathrm{HL}$ $(\mathrm{SD}=14 \mathrm{~dB})$. Based on the pure-tone average for the better ear, 66\% (293) of patients had no hearing loss, $29 \%$ (128) had mild hearing loss, and 5\% (23) had moderate hearing loss. Based on the pure-tone average for the worse ear, $49 \%$ of patients (217) had no hearing loss, $37 \%$ (163) had mild hearing loss, $13 \%$ (58) had moderate hearing loss, $0.7 \%$ (3) had severe hearing loss, and $0.7 \%$ (3) had profound hearing loss.

Based on the values of ULLmin, 30\% (133) of patients had ULLs $\leq 77 \mathrm{~dB}$ HL, indicating hyperacusis. Four percent (18) of patients were diagnosed with severe hyperacusis, as indicated by ULLs $\leq 30 \mathrm{~dB}$ for at least one of the measured frequencies in at least one ear.

\section{Relation between Insomnia and Tinnitus}

There was a significant association between the THI category and ISI category; $\chi_{(9)}^{2}=137, p<0.001$. Patients in the moderate and severe tinnitus handicap categories were more likely to have moderate or severe insomnia. 
Table 1. Means and SDs of Scores of the Study Population for the ISI, HADS, THI, VAS, and HQ

\begin{tabular}{lcc}
\hline Questionnaire & Mean & SD \\
\hline ISI (0-28) & 12.3 & 7.2 \\
HADS (depression) (0-21) & 6.0 & 4.6 \\
HADS (anxiety) (0-21) & 8.8 & 4.7 \\
THI (0-100) & 45 & 24 \\
VAS (tinnitus loudness) (0-10) & 6.0 & 2.0 \\
VAS (tinnitus annoyance) (0-10) & 6.2 & 2.5 \\
VAS (effect of tinnitus on life) (0-10) & 5.1 & 2.8 \\
HQ (0-42) & 17.6 & 9.1 \\
\hline
\end{tabular}

\section{Relation between Insomnia and Anxiety and Depression}

There was a significant association between the HADS anxiety category and ISI category; $\chi_{(6)}^{2}=107$, $p<0.001$. Patients with abnormal anxiety were more likely to have moderate and severe insomnia.

There was a significant association between the HADS depression category and ISI category; $\chi_{(6)}^{2}=125$, $p<0.001$. Patients with abnormal depression were more likely to have severe insomnia.

\section{Insomnia and Hyperacusis}

The mean ISI score was $14.2(\mathrm{SD}=7.3)$ for patients with tinnitus combined with hyperacusis (diagnosed based on ULLmin values) compared with 11.5 (SD = 7.1) for patients with tinnitus only, and the difference was significant ( $p=0.0003)$. The mean ISI score was 15.5 ( SD = 7.1) for patients with tinnitus combined with hyperacusis (diagnosed based on HQ scores) compared with 10.9 (SD = 6.8) for patients with tinnitus only, and the difference was significant $(p<0.001)$. The mean ISI score was 15.3 ( $\mathrm{SD}=6.9$ ) for patients with tinnitus combined with severe hyperacusis (diagnosed based on ULL values) compared with $12.2(\mathrm{SD}=7.2)$ for patients with tinnitus without severe hyperacusis. However, this difference was not significant ( $p=0.078)$. Overall, these results indicate that sleep problems tend to be worse for patients with tinnitus combined with hyperacusis than for patients with tinnitus only.

\section{Factors Related to Insomnia}

There were significant correlations between ISI scores and the following variables: THI scores $(r=0.58, p<$ $0.001)$, VAS scores for tinnitus annoyance $(r=0.47$, $p<0.001)$, VAS scores for tinnitus loudness $(r=0.36$, $p<0.001$ ), VAS scores for effect of tinnitus on life $(r=0.45, p<0.001)$, HADS anxiety and depression scores $(r=0.53$ and $r=0.57$, respectively, both $p<$ $0.001)$, HQ scores $(r=0.39, p<0.001)$, and ULLmin values $(r=-0.18, p=0.0001)$. There were no significant correlations between ISI scores and the pure-tone average for the better ears $(r=0.04, p=0.4)$ or the worse ears $(r=-0.005, p=0.91)$, and age $(r=-0.01$, $p=0.8)$. There was no significant difference in ISI scores between males and females $(p=0.83)$.

All variables that were significantly correlated with ISI scores were included in a stepwise linear-regression model. The following variables did not significantly contribute to the prediction of ISI scores: VAS for tinnitus loudness $(p=0.87)$, VAS for effect of tinnitus on life $(p=0.77)$, HQ score $(p=0.62)$, and ULLmin ( $p=$ 0.057). These variables were eliminated from the model. As shown in Table 3, in the final model, ISI scores were significantly associated with HADS depression scores, tinnitus annoyance as measured via the VAS, HADS

Table 2. Means (SD) of HTs (dB HL) and ULLs for Each Ear of the Study Population

\begin{tabular}{|c|c|c|c|c|c|c|}
\hline & \multicolumn{6}{|c|}{ Frequency, kHz } \\
\hline & 0.25 & 0.5 & 1 & 2 & 4 & 8 \\
\hline \multirow[t]{2}{*}{ HT right } & $17(14)$ & $17(14)$ & $18(16)$ & $20(18)$ & $31(22)$ & $38(27)$ \\
\hline & $\mathrm{n}=444$ & $\mathrm{n}=444$ & $\mathrm{n}=444$ & $\mathrm{n}=444$ & $\mathrm{n}=444$ & $\mathrm{n}=442$ \\
\hline \multirow[t]{2}{*}{ HT left } & $16(13)$ & $17(14)$ & $17(14)$ & $20(17)$ & $32(21)$ & $39(25)$ \\
\hline & $n=444$ & $n=444$ & $n=444$ & $n=444$ & $n=444$ & $n=443$ \\
\hline \multirow[t]{2}{*}{ ULL right } & 83 (15) & $84(15)$ & $85(14)$ & 83 (16) & $85(16)$ & 81 (19) \\
\hline & $n=443$ & $n=443$ & $n=443$ & $n=443$ & $n=438$ & $\mathrm{n}=435$ \\
\hline \multirow[t]{2}{*}{ ULL left } & $83(15)$ & $84(15)$ & $84(14)$ & $84(15)$ & $85(16)$ & $82(18)$ \\
\hline & $\mathrm{n}=442$ & $\mathrm{n}=442$ & $n=443$ & $n=443$ & $\mathrm{n}=441$ & $\mathrm{n}=437$ \\
\hline
\end{tabular}

Notes: Not all patients were tested at all frequencies. The number of patients for each cell is indicated by $\mathrm{n}$. 
anxiety scores, and THI scores. The model explained $45 \%$ of the variance in ISI scores.

\section{DISCUSSION}

$\mathrm{M}$ ore than two-thirds of patients with tinnitus and/ or hyperacusis who sought help from an audiology National Health Service clinic in the United Kingdom had insomnia as measured using the ISI. Although VAS scores for tinnitus loudness were significantly correlated with ISI scores, the VAS loudness scores did not significantly predict insomnia in the regression model. Rather, the model showed that insomnia was significantly related to tinnitus annoyance, depression and anxiety, and tinnitus handicap. The failure to find a significant effect of tinnitus loudness in the regression model may have occurred for two reasons: (a) the contribution of tinnitus loudness to variability in insomnia may be small compared with the contribution of other variables included in the model; (b) the relationship between tinnitus loudness and insomnia may be mediated via other variables, such as depression and anxiety and tinnitus annoyance (Aazh and Moore, 2019).

Several other researchers have reported a relationship between tinnitus loudness and sleep disturbance (Meikle et al, 1984; Folmer and Griest, 2000). Folmer and Griest (2000) measured sleep disturbance with just one question: "Does your tinnitus interfere with sleep?" They reported that there was a significant difference in tinnitus loudness between a group of patients who often had trouble sleeping and groups who never or sometimes had sleep disturbance $(p<0.001)$. Meikle et al (1984) reported that there was a statistically significant relationship between the severity of tinnitus and the proportion of people reporting sleep disturbance caused by their tinnitus. These researchers did not assess whether the relationship between sleep disturbance and tinnitus remained significant after taking into account the effect of other relevant variables, such as depression. Their results are consistent with our results because we also found a significant correlation between VAS scores for tinnitus loudness and ISI scores. However, our regression analysis suggested that the relationship is an indirect one rather than a causal one.

About $30 \%$ of our patients had hyperacusis based on ULLmin values, and $32 \%$ had hyperacusis as measured via the $\mathrm{HQ}$ in addition to tinnitus. Unfortunately, it was not recorded whether tinnitus or hyperacusis was the primary complaint for each patient. ISI scores were significantly higher for patients with tinnitus combined with hyperacusis than for patients with tinnitus alone. This is consistent with the results of Nordin and Nordin (2016), as described in the Introduction, who reported a higher incidence of insomnia among the general population for those who self-reported sound tolerance problems. Our results also showed that $\mathrm{HQ}$ scores and ULLmin values were significantly correlated with ISI scores $(r=0.39, p<0.001)$ and $(r=-0.18, p<$ $0.001)$, respectively. This is consistent with the results of Hebert and Carrier (2007), who reported a significant relationship between $\mathrm{HQ}$ scores and sleep disturbances measured via the PSQI.

Taken together, these results appear to support an association between hyperacusis and insomnia. However, the step-wise linear regression model showed that the measures of hyperacusis (HQ scores and ULLmin scores) did not significantly predict insomnia. As for tinnitus loudness, this is consistent with hyperacusis having an indirect effect on insomnia, mediated via depression and anxiety. Future research should explore the processes by which tinnitus loudness and hyperacusis may lead indirectly to insomnia.

Our final model, which explained $45 \%$ of the variance in ISI scores, showed that ISI scores were significantly predicted by tinnitus annoyance, depression, anxiety, and tinnitus handicap. Contrary to our results, Hebert and Carrier (2007) reported that insomnia scores measured via the PSQI were associated with $\mathrm{HQ}$ and BDI-II scores but not with tinnitus distress as measured via the TRQ. Their regression model explained $42.5 \%$ of the variance in PSQI scores. However, the two studies agree that depression is significantly associated with insomnia among patients with tinnitus. This is consistent with previous work showing a link between insomnia and depressive symptoms in patients with tinnitus (Cronlein et al, 2016; Xu et al, 2016; Aazh et al, 2017). Future studies should explore whether insomnia in people with tinnitus needs to be managed in conjunction with treatment for depression.

The discrepancy between our results and those of Hebert and Carrier (2007) with regard to the relationship between tinnitus-related measures and insomnia could be related to the following facts: (a) The sample size was smaller in their study $(\mathrm{n}=51)$ than in ours

Table 3. Results of the Stepwise Linear Regression Model to Determine Variables that Predict ISI Scores

\begin{tabular}{lcrrr}
\hline & Coefficient & $p$ Value & \multicolumn{2}{c}{ 95\% Confidence Interval } \\
\hline VAS for tinnitus annoyance & 0.49 & 0.001 & 0.21 & 0.77 \\
Depression subscale of HADS & 0.44 & $<0.001$ & 0.27 & 0.61 \\
Anxiety subscale of HADS & 0.21 & 0.012 & 0.045 & 0.37 \\
THI & 0.07 & $<0.001$ & 0.037 & 0.10 \\
\hline
\end{tabular}

Note: $\mathrm{n}=444$. 
$(\mathrm{n}=444)$, making their study more vulnerable to type II errors. (b) Their study was focused on older adults with a mean age of 68 years ( $\mathrm{SD}=6.5$ years), whereas our patients had a mean age of 54 years ( $\mathrm{SD}=15$ years). Insomnia is highly prevalent among older people, and tinnitus may not markedly increase age-related sleep difficulties (Aazh et al, 2017). (c) Hebert and Carrier (2007) modified the TRQ by removing one item that specifically referred to sleep disturbances. We used the unmodified THI, which also includes one item assessing the impact of tinnitus on sleep.

Another discrepancy across studies is that Hebert and Carrier (2007) found a significant relationship between HQ scores and insomnia scores, despite controlling for depression and tinnitus-related measures, whereas we did not. This may also be due to the difference in study populations. Hyperacusis may have a greater influence on insomnia among older than among younger patients. Further research is needed to assess whether this is the case.

Four percent of the patients in our study were diagnosed with severe hyperacusis, diagnosed as suggested by Aazh and Moore (2018) on the basis of ULLs $\leq 30 \mathrm{~dB}$ HL for at least one of the measured frequencies in at least one ear. Although the mean ISI was worse for patients with tinnitus combined with severe hyperacusis than for the remainder of the population, the difference was not significant. This could be due to the small number of patients who were diagnosed with severe hyperacusis, leading to a type II error. Future studies with larger sample sizes should explore whether severe hyperacusis is related to insomnia.

\section{CONCLUSIONS}

$\mathrm{F}$ or patients who sought help concerning tinnitus and/or hyperacusis in a UK National Health Service audiology clinic, a stepwise multiple-regression analysis showed that insomnia was significantly predicted by tinnitus annoyance, depression, anxiety, and tinnitus handicap. Insomnia was not significantly predicted by VAS scores for tinnitus loudness and effect of tinnitus on life, scores for hyperacusis handicap, ULLmin values, and the pure-tone average of the better and worse ears, age, or gender.

\section{REFERENCES}

Aazh H, Lammaing K, Moore BCJ. (2017) Factors related to tinnitus and hyperacusis handicap in older people. Int J Audiol 56: 677-684

Aazh H, Moore BCJ. (2017) Factors related to uncomfortable loudness levels for patients seen in a tinnitus and hyperacusis clinic. Int J Audiol 56:793-800.

Aazh H, Moore BCJ. (2018) Prevalence and characteristics of patients with severe hyperacusis among patients seen in a tinnitus and hyperacusis clinic. J Am Acad Audiol 29:626-633.
Aazh H, Moore BCJ. (2019) Tinnitus loudness and the severity of insomnia: a mediation analysis. Int $J$ Audiol 58:208212.

Aazh H, Moore BCJ, Lammaing K, Cropley M. (2016) Tinnitus and hyperacusis therapy in a UK National Health Service audiology department: patients' evaluations of the effectiveness of treatments. Int $J$ Audiol 55:514-522.

American Psychiatric Association. (2000) Diagnostic and Statistical Manual of Mental Disorders: DSM-IV-TR ${ }^{\circledR}$. Washington, DC: American Psychiatric Association Publishing.

Andersson G, Edvinsson E. (2008) Mixed feelings about living with tinnitus: a qualitative study. Audiol Med 6:48-54.

Baguley DM. (2018) Reflections on the association between tinnitus and hyperacusis. In: Fagelson M, Baguley DM, eds. Hyperacusis and Disorders of Sound Tolerance. San Diego, CA: Plural.

Baliatsas C, van Kamp I, Swart W, Hooiveld M, Yzermans J. (2016) Noise sensitivity: symptoms, health status, illness behavior and co-occurring environmental sensitivities. Environ Res 150: 8-13.

Bastien CH, Vallieres A, Morin CM. (2001) Validation of the insomnia severity index as an outcome measure for insomnia research. Sleep Med 2:297-307.

British Society of Audiology (BSA). (2011a) Pure-Tone AirConduction and Bone-Conduction Threshold Audiometry with and without Masking: Recommended Procedure. Reading, UK: British Society of Audiology.

British Society of Audiology (BSA). (2011b) Recommended Procedure: Determination of Uncomfortable Loudness Levels. Reading, UK: British Society of Audiology.

Buysse DJ, Reynolds CF, 3rd, Monk TH, Berman SR, Kupfer DJ. (1989) The Pittsburgh Sleep Quality Index: a new instrument for psychiatric practice and research. Psychiatry Res 28: 193-213.

Cronlein T, Langguth B, Popp R, Lukesch H, Pieh C, Hajak G, Geisler P. (2013) Regensburg Insomnia Scale (RIS): a new short rating scale for the assessment of psychological symptoms and sleep in insomnia; study design: development and validation of a new short self-rating scale in a sample of 218 patients suffering from insomnia and 94 healthy controls. Health Qual Life Outcomes 11:65.

Cronlein T, Langguth B, Pregler M, Kreuzer PM, Wetter TC, Schecklmann M. (2016) Insomnia in patients with chronic tinnitus: cognitive and emotional distress as moderator variables. $J$ Psychosom Res 83:65-68.

Fioretti AB, Fusetti M, Eibenstein A. (2013) Association between sleep disorders, hyperacusis and tinnitus: evaluation with tinnitus questionnaires. Noise Health 15:91-95.

Foley DJ, Monjan AA, Brown SL, Simonsick EM, Wallace RB, Blazer DG. (1995) Sleep complaints among elderly persons: an epidemiologic study of three communities. Sleep 18:425-432.

Folmer RL, Griest SE. (2000) Tinnitus and insomnia. Am J Otolaryngol 21:287-293.

Hallam RS, Jakes SC, Hinchcliffe R. (1988) Cognitive variables in tinnitus annoyance. Br J Clin Psychol 27(Pt 3):213-222.

Hebert S, Carrier J. (2007) Sleep complaints in elderly tinnitus patients: a controlled study. Ear Hear 28:649-655. 
Khalfa S, Dubal S, Veuillet E, Perez-Diaz F, Jouvent R, Collet L. (2002) Psychometric normalization of a hyperacusis questionnaire. ORL J Otorhinolaryngol Relat Spec 64:436-442.

Kutner MH, Nachtsheim C, Neter J. (2004) Applied Linear Regression Models. 4th ed. New York, NY: McGraw-Hill Education.

Lasisi AO, Gureje O. (2011) Prevalence of insomnia and impact on quality of life among community elderly subjects with tinnitus. Ann Otol Rhinol Laryngol 120:226-230.

Meikle MB, Vernon J, Johnson RM. (1984) The perceived severity of tinnitus. Some observations concerning a large population of tinnitus clinic patients. Otolaryngol Head Neck Surg 92:689-696.

Miguel GS, Yaremchuk K, Roth T, Peterson E. (2014) The effect of insomnia on tinnitus. Ann Otol Rhinol Laryngol 123:696-700.

Newman CW, Jacobson GP, Spitzer JB. (1996) Development of the tinnitus handicap inventory. Arch Otolaryngol Head Neck Surg 122:143-148.

Nordin M, Nordin S. (2016) Sleep and sleepiness in environmental intolerances: a population-based study. Sleep Med 24:1-9.

Park J, Chung S, Lee J, Sung JH, Cho SW, Sim CS. (2017) Noise sensitivity, rather than noise level, predicts the non-auditory effects of noise in community samples: a population-based survey. BMC Public Health 17:315.

Schecklmann M, Pregler M, Kreuzer PM, Poeppl TB, Lehner A, Crönlein T, Wetter TC, Frank E, Landgrebe M, Langguth B. (2015) Psychophysiological associations between chronic tinnitus and sleep: a cross validation of tinnitus and insomnia questionnaires. Biomed Res Int 2015:461090.

Steer RA, Ball R, Ranieri WF, Beck AT. (1997) Further evidence for the construct validity of the Beck depression Inventory-II with psychiatric outpatients. Psychol Rep 80:443-446.

Tyler RS, Baker JL. (1983) Difficulties experienced by tinnitus sufferers. J Speech Hear Disord 48:150-154.

Visser P, Hofman WF, Kumar A, Cluydts R, de Diana IPF, Marchant P, Bakker HJ, van Diest R, Poelstra PAM. (1978) Sleep and mood: measuring the sleep quality. In: Priest RG, Pletscher A, Ward J, eds. Proceedings of the Northern European Symposium on Sleep Research. Basel, Lancaster: MTP Press Limited, 135-145.

Wilson PH, Henry J, Bowen M, Haralambous G. (1991) Tinnitus reaction questionnaire: psychometric properties of a measure of distress associated with tinnitus. J Speech Hear Res 34:197-201.

Xu Y, Yao J, Zhang Z, Wang W. (2016) Association between sleep quality and psychiatric disorders in patients with subjective tinnitus in China. Eur Arch Otorhinolaryngol 273:3063-3072.

Zigmond AS, Snaith RP. (1983) The hospital anxiety and depression scale. Acta Psychiatr Scand 67:361-370.

Zung WW. (1971) A rating instrument for anxiety disorders. Psychosomatics 12:371-379.

Zung WW. (1974) The measurement of affects: depression and anxiety. Mod Probl Pharmacopsychiatry 7:170-188. 